\title{
ONCOCYTOMA OF THE NASAL CAVITY
}

\author{
Singh A K, Agrahari K N, Baitha $S^{*}$ \\ Sharma R*, Reddy N S*, Talwar O P*
}

\section{ABSTRACT}

Oncocytoma of the nasal cavity is an extremely rare condition with only a few cases reported in the literature. A case of oncocytoma of the nasal cavity in a 12 years old Nepali boy is presented along with a brief review of the relevant literature.

Key Words: Oncocytoma, Oxyphil adenoma, Nasal cavity.

\section{INTRODUCTION}

Oncocytoma (oxyphil adenoma) is a rare tumour of epithelial origin accounting for less than $1 \%$ of all salivary gland tumours. They are usually benign and arise from oncocytes. Majority of them occur in the parotid gland. They are also found in the pancrease, thyroid, parathyroid, pituitary, adrenal glands, kidney and respiratory tract. ${ }^{1}$ The nasal cavity is an extremely rare site for occurrence of this tumour with only a few cases reported in the literature. ${ }^{2}$ We report a case of oncocytoma of the nasal cavity which to our knowledge from medline search is the $16^{\text {th }}$ case reported in the literature and the $1^{\text {st }}$ case reported from Nepal.

\section{CASE REPORT}

A 12 years old Nepali boy presented at the department of ENT, Western Regional Hospital, Pokhara, with history of left nasal obstruction, blood stained nasal discharge and occasional epistaxis for 1 year. Examination revealed a smooth, lobulated, grayish white, polypoidal mass filling up the left nasal cavity pushing the septum to the right side. The mass was insensitive, soft, non-friable and did not bleed on touch. There was no cervical lymphadenopathy. His haemoglobin was $14.0 \mathrm{gm} / \mathrm{dl}$, total count was $6000 / \mathrm{cmm}$ and differential count was Neutrophil 60\%, Lymphocyte 35\% and Eosinophil 5\%. Urine analysis was normal. Plain x-ray occipitomental view of the paranasal sinuses showed soft tissue shadow in the left nasal cavity with normal sinuses and bony outlines, Plain x-ray lateral view of the nasopharynx was unremarkable. Examination under general anaesthesia revealed the mass arising from the lateral wall of the left nasal cavity above the middle turbinate. A complete pernasal excision of the mass was done. Bleeding was minimal and controlled by unilateral anterior nasal pack, which was removed after 24 ours. Thepatient had an uneventful recovery and was discharged on the $2^{\text {d }}$ post-operative day.

The mass measured $3.5 \times 3 \times 2$ cms. Histological examination revealed an oncovytoma (Fig. 1\&2). The tumour was made up of sheets, nests and papillary structures of large polygonal cells

* Manipal Teaching Hospital, Pokhara, Nepal.

Address for correspondence : $\quad$ Dr. A. K. Singh

Dept. of Otorhinolaryngology - HNS,

Manipal Teaching Hospital, Post Box No.: 341

Phulbari, Pokhara - 33701, Nepal

Email: kennedyakoijam@rediffmail.com

.INMA .lanılarv - Fehrı Iary 20ก.3 4? 
Fig. 1

Fig. 1: Microphotograph of oncocytoma of nasal cavity (H\&EX20).

Fig. 2

Fig.2: Microphotograph of oncocytoma of nasal cavity (H\&EX100)

having abundant eosinophillic granular cytoplasm and large central vesicular nuclei. There was moderate nuclear pleomorphism and occasional mitotic figures. A partial capsule made up of thick collagen bundles was also observed.

The patient was reviewed one week after surgery and there was no evidence of residual disease. At 9 months after surgery he was asymtomatic without recurrence.

\section{DISCUSSION}

The term 'oncocyte' derived from the Greek word 'onkousthai' which means 'swollen' was coined by Hamperl in 1931 to describe a type of cell with swollen eosinophilic granular cytoplasm..$^{3,4}$ In 1932 Jaffe introduced the term 'oncocytoma' to designate a salivary gland tumour made up of these swollen cells. ${ }^{2,5}$ The histogenesis of oncocytoma is still uncertain., ${ }^{2,3,4}$ They probably represent neoplastic transformation of oncocytic cell which is now considered an epithelial cell with mitochondrial hyperplasia. A functional defect in the oxidative phosphorylation process seems to be the major cause of mitochondrial hyperplasia. ${ }^{2}$ Oncocytic cells may be found in many organs and probably represents a form of degeneration, regression, ageing or burnout of a cell with loss of its specific function. However outside the endocrine glands, kidneys and major salivary glands, oncovytomas are unusual and the localization in the nasal cavity is exceptional., ${ }^{2,5}$

Oncocytoma of the nasal cavity is usually seen in elderly patients and rarely before the age of 20 years. The usually present with epistaxis, nasal obstruction and rhinorrhoea. ${ }^{2,3,5}$ The lesion can occur anywhere in the nasal cavity ranging from the nasal septum to the lateral nasal wall..$^{5}$ Our patient is probably the youngest reported in the literature and he presented with the usual symptoms described in the literature.

Most oncocytomas are benign. 3,5 However their clinical behavior cannot be predicted by their morphological characteristics (cytological atypia, mitotic activity) but rather by their site of origin and local growth. ${ }^{2,5}$ The malignant potential of those arising in the nasal cavity is mostly due to their locally aggressive nature. ${ }^{2,3,5}$ Diagnosis of malignancy in these tumours depend on the presence of frequent mitosis and cellular pleomorphism with invasion and destruction of adjacent structures; perineural, intravascular or lymphatic invasion; regional and distant metastasis. ${ }^{3,5}$ These features were not observed in our patient.

Surgical excision is the mainstay in the treatment of oncocytoma. However combined modality-surgery followed by radiation therapy has been used to treat the malignant variety. ${ }^{3}$ Considering the rarity of nasal oncocytoma and unpredictability of their clinical course a long term follow up is recommended.

\section{REFERENCE}

1. Suen J Y, Snyderman NL. Benign neoplasms of the salivary glands. In: Cummings CW, ed. Otolaryngology-Headand Neck Surgery. 2nded. St Louis: Mosby-YearBook Inc, 1993; 10291042.

2. Comin CE, Dini M, Russo GL. Oncocytoma of the nasal cavity: report of a case and review of the literature. The journal of Laryngology and Otology 1997; 111: 671-673.

3. NayakDR, PillaiS, BalakrishnanR, Thomas R, RaoR. Malignant oncocytoma of thenasal cavity: a casereport AmericanJ oumal ofOtolaryngology 1999; 20: 323-327.

4. Colreavy MP, SigstonE, LacyPD, BalasubramaniamGS, Lyons BM. Post-nasal space oncocytoma: a different approach to a rare tumour. The J ournal of Laryngology and Otology 2001; 115: 57-59.

5. HamdanAL, Kahwagi G, FarhatF, Tawii A. Oncocytoma of the nasal septum: a rare cause ofepistaxis. Otolaryngology-Head and Neck Surgery 2002; 126: 440-441. 